\title{
A total variation-based reconstruction method for dynamic MRI
}

\author{
GERMANA LANDI*, ELENA LOLI PICCOLOMINI $\dagger$ and FABIANA ZAMA \\ Department of Mathematics, Piazza Porta S. Donato 5, Bologna, Italy
}

(Received 31 January 2007; in final form 27 November 2007)

\begin{abstract}
In recent years, total variation (TV) regularization has become a popular and powerful tool for image restoration and enhancement. In this work, we apply TV minimization to improve the quality of dynamic magnetic resonance images. Dynamic magnetic resonance imaging is an increasingly popular clinical technique used to monitor spatio-temporal changes in tissue structure. Fast data acquisition is necessary in order to capture the dynamic process. Most commonly, the requirement of high temporal resolution is fulfilled by sacrificing spatial resolution. Therefore, the numerical methods have to address the issue of images reconstruction from limited Fourier data. One of the most successful techniques for dynamic imaging applications is the reduced-encoded imaging by generalized-series reconstruction method of Liang and Lauterbur. However, even if this method utilizes a priori data for optimal image reconstruction, the produced dynamic images are degraded by truncation artifacts, most notably Gibbs ringing, due to the spatial low resolution of the data. We use a TV regularization strategy in order to reduce these truncation artifacts in the dynamic images. The resulting TV minimization problem is solved by the fixed point iteration method of Vogel and Oman. The results of test problems with simulated and real data are presented to illustrate the effectiveness of the proposed approach in reducing the truncation artifacts of the reconstructed images.
\end{abstract}

Keywords: Total variation; Regularization; Magnetic resonance imaging; Image reconstruction

\section{Introduction}

Magnetic resonance imaging (MRI) is a valuable non-invasive diagnostic tool used in medicine for acquiring cross sectional images of the human body. Dynamic MRI is an emerging application of MRI which allows to study changes over time in the tissue structure.

For example, dynamic MRI is used in dynamic contrast-enhancement or functional brain studies, cardiac imaging and real-time monitoring of surgical interventions. Typically, in dynamic applications, a temporal series of Magnetic Resonance (MR) images of the same slice of the imaged structure is acquired. In order to capture and study the dynamic process both high temporal and spatial resolutions are required. Unfortunately, the technological and physiological limits on the MR technique make difficult to simultaneously fulfill these requirements. In the past years, several methods have been proposed with the common aim to reduce the data acquisition time. Among others, the so-called reduced encodings methods

\footnotetext{
*Corresponding author. Email: landig@dm.unibo.it

†Email: piccolom@dm.unibo.it

†Email: zama@dm.unibo.it 
speed up the acquisition time by acquiring a time series of reduced dynamic data sets and one high resolution reference data set which is collected before the dynamic process. MRI is by its nature a Fourier encoded modality: the data are collected in the $k$-space, a frequency $2 \mathrm{D}$ domain whose principal directions are called frequency-encoded direction $\left(k_{x}\right)$ and phaseencoded direction $\left(k_{y}\right)$. The dynamic data sets consist of a small and central part (a $k$-hole) of the $k$-space constituted by the low spatial frequencies along the phase-encoded direction. The rationale for truncating the dynamic data lies in the fact that the morphological details are mainly encoded by the high frequencies while the dynamic information is mainly contained in the low frequency part of the $k$-space. Therefore, assuming that during the dynamic process no significant changes occur in the underlying morphology, the dynamic variation can be characterized by repeated sampling of the central $k$-hole. The reference data set provides the a priori information concerning the high frequencies uncollected during the dynamic process. Usually, the MR images are reconstructed by using a 2D discrete inverse Fourier transform (2DIFT) of the data. In the case of reduced encodings dynamic MRI, the image reconstruction problem from incomplete Fourier data is a difficult inverse problem, since the images obtained by a 2DIFT of the dynamic data suffer from the well-known truncation artifacts including ringing and blurring. In this case, the reconstruction methods obtain high resolution dynamic images with the use of the high resolution reference image. One of the most successful reconstruction techniques is the reduced-encoded imaging by generalized-series reconstruction (RIGR) method of Liang and Lauterbur [10]. In this method, the unknown dynamic MR images are represented by means of a parametric model embedding the a priori information deriving from the reference image. However, due to the partial encode of the data, also the dynamic images obtained with the RIGR technique exhibit truncation artifacts degrading their quality and compromising their use in clinical applications. In this work, we propose to include in the RIGR method a total variation (TV)based regularization strategy in order to reduce the damaging artifacts and improve the quality of the reconstructed images. TV regularization is a popular approach used in image processing for reducing noise and blur in images while preserving sharp edges [11]. Moreover, TV regularization has been successfully used in some medical imaging applications such as positron emission tomography [4], single photon emission computed tomography [12] and diffraction ultrasound tomography [2]. In Ref.[5], TV regularization has been applied to MR images for image enhancement and noise removal. Recently, the authors have used TV regularization for post-processing the dynamic MR images obtained with Keyhole method [7,9]. The Keyhole technique belongs to the class of the reduced encodings methods and differs from the RIGR method in how the a priori information is incorporated into the imaging process. It has been shown by comparison [10] that the RIGR method gives improvements in image resolution over the Keyhole technique. The good performance of TV regularization with the Keyhole method motivates its use for improving the quality of the RIGR images. TV regularization entails an unconstrained minimization problem with highly nonlinear Euler-Lagrange equations. We solve these equations by the fixed point (FP) iteration method of Vogel and Oman [14,15] which is one of the most efficient and robust methods proposed in the literature for TV minimization. The main contribution of the paper therefore lies in the application and investigation of the TV regularization in order to improve the quality of the dynamic MR images obtained with the RIGR technique. The sequel is organized as follows. A review of the RIGR method is given in section 2 and the proposed TV-based RIGR method is presented in section 3. Experimental results and implementation details are described in section 4. Finally, conclusions are given in section 5 . 


\section{The classical RIGR method}

\subsection{Data acquisition and image formation}

In spin-echo MR experiments, the $k$-space is built-up row-wise and the raw data are sampled on a $2 \mathrm{D}$ rectangular trajectory as shown in figure 1 . Let $\Omega$ be the grid of points fully covering the $k$-space:

$$
\Omega=\left\{\left(n \Delta k_{x}, m \Delta k_{y}\right) \mid n=-N / 2, \ldots, N / 2-1, m=-M / 2, \ldots, M / 2-1\right\}
$$

where $\Delta k_{x}$ and $\Delta k_{y}$ are sampling intervals. Let $D\left(n \Delta k_{x}, m \Delta k_{y}\right)$ be the $k$-space datum acquired at the grid point $\left(n \Delta k_{x}, m \Delta k_{y}\right)$; the detected data form a $N \times M$ matrix $D\left(\mathbf{k}_{x}, \mathbf{k}_{y}\right)$. Let $I(\mathbf{x}, \mathbf{y})$ be the $N \times M$ image reconstructed by a 2 DIFT of the data matrix $D\left(\mathbf{k}_{x}, \mathbf{k}_{y}\right)$ :

$$
I(\mathbf{x}, \mathbf{y})=2 \operatorname{DIFT}\left(D\left(\mathbf{k}_{x}, \mathbf{k}_{y}\right)\right) .
$$

The final MR image that is represented is the magnitude of $I(\mathbf{x}, \mathbf{y})$.

In a fast dynamic spin-echo MRI experiment a fully encoded $N \times M$ reference data set $D_{\mathrm{R}}\left(\mathbf{k}_{x}, \mathbf{k}_{y}\right)$ is acquired before the dynamic process to provide the information on the outer $k$-space region uncollected during the dynamic process. The reference image $I_{\mathrm{R}}(\mathbf{x}, \mathbf{y})$ is reconstructed by a 2DIFT from $D_{\mathrm{R}}\left(\mathbf{k}_{x}, \mathbf{k}_{y}\right)$.

During the dynamic process the imaging time is decreased by collecting only the low spatial frequencies in the phase-encoded direction. Let $\Omega_{\text {low }}$ be the grid points of the low sampled $k$-space:

$$
\begin{aligned}
\Omega_{\mathrm{low}}= & \left\{\left(n \Delta k_{x}, m \Delta k_{y}\right) \mid n=-N_{\text {low }} / 2, \ldots, N_{\text {low }} / 2-1,\right. \\
& \left.m=-M / 2, \ldots, M / 2-1, N_{\text {low }}<<N\right\} .
\end{aligned}
$$

A sequence of low sampled $N_{\text {low }} \times M$ data matrices $\tilde{D}_{t}\left(\mathbf{k}_{x}, \mathbf{k}_{y}\right)$ defined as

$$
\left(\tilde{D}_{t}\left(\mathbf{k}_{x}, \mathbf{k}_{y}\right)\right)_{n, m}=\tilde{D}_{t}\left(n \Delta k_{x}, m \Delta k_{y}\right), \quad\left(n \Delta k_{x}, m \Delta k_{y}\right) \in \Omega_{\text {low }}, \quad t=1, \ldots, T,
$$

is acquired at $T$ successive time instants.

The acquired dynamic data sets $\tilde{D}_{t}\left(\mathbf{k}_{x}, \mathbf{k}_{y}\right), t=1, \ldots, T$, are inverse Fourier transformed along the fully encoded horizontal direction in order to obtain $\tilde{D}_{t}\left(\mathbf{k}_{x}, \mathbf{y}\right)$. Since, the number of phase-encodings is reduced from $N$ to $N_{\text {low }}$, performing a DIFT along the vertical direction produces images with evident truncation artifacts. A reconstruction method should provide
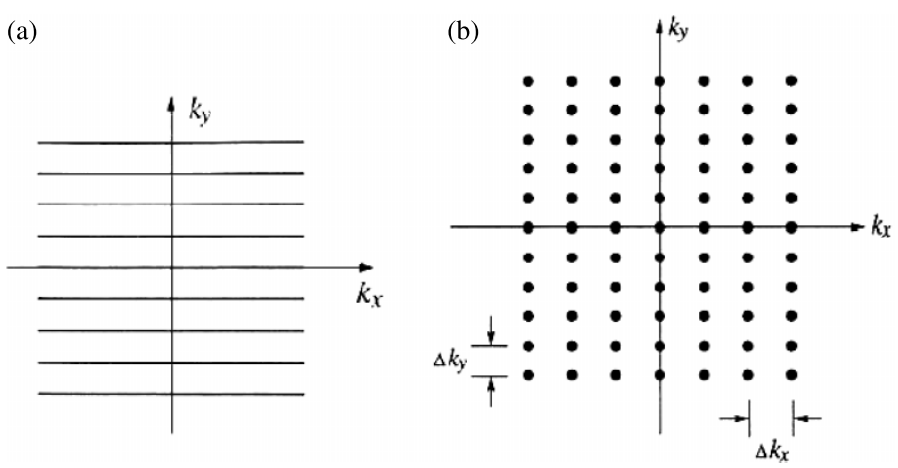

Figure 1. $\quad k$-space sampling; (a) sampling trajectory; (b) raw data matrix. 
good quality high-resolution images. A crucial point is that the high resolution dynamic image can be obtained column-wise from $\tilde{D}_{t}\left(\mathbf{k}_{x}, \mathbf{y}\right)$ by independently reconstructing each column. In this way, the problem is reduced to the reconstruction of $M$ signals of size $N$. Therefore, in the sequel, we will present the RIGR method only in the 1D case. For easier notation, in the following we omit the temporal index $t$ and we call $\mathbf{k}=\left(-\left(N_{\text {low }} / 2\right) \Delta k, \ldots\right.$, $\left.\left(N_{\text {low }} / 2-1\right) \Delta k\right)$ and $\mathbf{x}=(0, \Delta x, \ldots,(N-1) \Delta x)$ the point vectors in the data and in the image domain, respectively.

\subsection{The reconstruction method}

The RIGR method [10] belongs to the class of the model-based reduced encodings method which are described by a common equation $[6,8]$. According to this common equation, the unknown dynamic signal $I(\mathbf{x})$ can be factorized as:

$$
I(\mathbf{x})=I_{*}(\mathbf{x}) * I_{\mathrm{d}}(\mathbf{x})
$$

where $I_{*}(\mathbf{x})$ is the multiplicative factor built into the model (1) for dynamic MRI and .* is the element-wise product. The dynamic factor $I_{\mathrm{d}}(\mathbf{x})$ represents the dynamic features of $I(\mathbf{x})$ lacking in $I_{*}(\mathbf{x})$. This function is represented by a parametric model as

$$
I_{\mathrm{d}}(\mathbf{x})=\sum_{\ell=-N_{\text {low }} / 2}^{N_{\text {low }} / 2-1} \alpha_{\ell} \phi_{\ell}(\mathbf{x}) \text {. }
$$

The number of terms of the sum is determined by the number of available information on the desired dynamic signal, i.e. the number of acquired $k$-space dynamic data. By substituting the representation (2) for $I(\mathbf{x})$, the equation (1) becomes:

$$
I(\mathbf{x})=I_{*}(\mathbf{x}) \cdot * \sum_{\ell=-N_{\text {low }} / 2}^{N_{\text {low }} / 2-1} \alpha_{\ell} \phi_{\ell}(\mathbf{x}) .
$$

Equation (3) describes the wide class of the model-based reduced encodings methods where every dynamic signal $I(\mathbf{x})$ is uniquely determined by the model parameters $\alpha_{\ell}, \ell=-N_{\text {low }} /$ $2, \ldots, N_{\text {low }} / 2-1$.

In this way, the problem of reconstructing the high resolution dynamic signal $I(\mathbf{x})$ from the low resolution dynamic data set $\tilde{D}(\mathbf{k})$ is converted to a coefficients estimation problem. Specifically, let us consider the low resolution version $\tilde{I}(\mathbf{x})$ of $I(\mathbf{x})$ computed at the points $\left(0, \Delta \tilde{x}, \ldots\left(N_{\text {low }}-1\right) \Delta \tilde{x}\right)$; for the signal $\tilde{I}(\mathbf{x})$ the equation (1) becomes:

$$
\tilde{I}(\mathbf{x})=\tilde{I}_{*}(\mathbf{x}) \cdot * \tilde{I}_{\mathrm{d}}(\mathbf{x})
$$

where $\tilde{I}_{*}(\mathbf{x})$ is the low resolution version of the multiplicative factor. By applying the DFT to both the terms of (4), we obtain the expression

$$
\tilde{D}(\mathbf{k})=\tilde{D}_{*}(\mathbf{k}) \otimes \tilde{D}_{\mathrm{d}}(\mathbf{k}),
$$

where $\otimes$ represents the convolution product. The convolution product in (5) can be represented in matrix form

$$
\tilde{D}_{*}(\mathbf{k}) \otimes \tilde{D}_{\mathrm{d}}(\mathbf{k})=\mathcal{H} \tilde{D}_{\mathrm{d}}(\mathbf{k})
$$


where $\mathcal{H}$ is a $N_{\text {low }} \times N_{\text {low }}$ matrix with a block Toeplitz structure. Hence:

$$
\mathcal{H} \tilde{D}_{\mathrm{d}}(\mathbf{k})=\tilde{D}(\mathbf{k}) .
$$

Since, the matrix $\mathcal{H}$ is ill-conditioned, a regularized solution of the linear system is computed by means of the Lavrent'ev regularization method, i.e. the following linear system is solved:

$$
(\mathcal{H}+\gamma 1) \tilde{D}_{\mathrm{d}}(\mathbf{k})=\tilde{D}(\mathbf{k})
$$

where $\gamma>0$ is the regularization parameter and 1 is the identity matrix.

The RIGR method uses a set of $N_{\text {low }}$ complex exponential basis functions defined as

$$
\phi_{\ell}(x)=\mathrm{e}^{2 \pi \sqrt{-1}\left(k_{\ell} x\right)}, \quad \ell=-N_{\text {low }} / 2, \ldots, N_{\text {low }} / 2-1
$$

and the multiplicative factor is the reference image: $I_{*}(\mathbf{x})=I_{\mathrm{R}}(\mathbf{x})$. In this case, the coefficient vector $\boldsymbol{\alpha}=\left(\alpha_{-N_{\mathrm{low}} / 2}, \ldots, \alpha_{N_{\mathrm{low}} / 2-1}\right)^{t}$ is simply computed from (2) by:

$$
\boldsymbol{\alpha}=\operatorname{DFT}\left(I_{\mathrm{d}}(\tilde{\mathbf{x}})\right)=\tilde{D}_{\mathrm{d}}(\mathbf{k})
$$

and $I_{\mathrm{d}}(\mathbf{x})$ is obtained by applying a DIFT to the zero-filled spectrum:

$$
I_{\mathrm{d}}(\mathbf{x})=\operatorname{DIFT}\left(Z P\left(\tilde{D}_{\mathrm{d}}(\mathbf{k})\right)\right),
$$

where $Z P\left(\tilde{D}_{\mathrm{d}}(\mathbf{k})\right)$ indicates the zero-padded $N \times 1$ data vector.

The described procedure is applied for the reconstruction of each column of each dynamic image of the sequence. The algorithm of the RIGR method for the reconstruction of a sequence of dynamic images is described in a Matlab-like language as follows.

\section{Algorithm 2.01.}

Input: Dynamic under-sampled data $\tilde{D}(:,:, t), t=1, \ldots, T$ of size $N_{\text {low }} \times M$. Reference image $I_{\mathrm{R}}$ of size $N \times M$.

Output: Dynamic RIGR images $I(:,:, t), t=1, \ldots, T$ of size $N \times M$.

$$
\begin{aligned}
& \text { for } t=1: T \\
& \quad \text { for } j=1: M
\end{aligned}
$$

Step 1: Compute the spectra $\tilde{D}_{*}(:, j, t)$ and the matrix $\mathcal{H}$.

Step 2: Compute $\tilde{D}_{\mathrm{d}}(:, j, t)$ by solving the linear system

$$
(\mathcal{H}+\gamma 1) \tilde{D}_{\mathrm{d}}(:, j, t)=\tilde{D}(:, j, t) .
$$

Step 3: Compute the high resolution dynamic factor $I_{\mathrm{d}}(:, j, t)$ :

$$
I_{\mathrm{d}}(:, j, t)=\operatorname{DIFT}\left(Z P\left(\tilde{D}_{\mathrm{d}}(:, j, t)\right) .\right.
$$

Step 4: Compute the high resolution dynamic signal $I(:, j, t)$ :

$$
I(:, j, t)=I_{\mathrm{R}}(:, j) * I_{\mathrm{d}}(:, j, t) .
$$




\section{The TV-regularized RIGR method}

Here, we propose a TV regularization approach to reduce the truncation artifacts arising from the RIGR method and to obtain images of improved quality. Let $I_{\mathrm{d}}$ be the RIGR dynamic factor obtained by (2). It is a $2 \mathrm{D}$ array of size $N \times M$, but it suffers from ringing artifacts due to the zero padding operation in (11). The RIGR dynamic factor $I_{\mathrm{d}}$ is regularized by solving the following unconstrained minimization problem:

$$
\min \mathcal{F}(I), \quad \mathcal{F}(I)=\frac{1}{2}\left\|I-I_{\mathrm{d}}\right\|_{2}^{2}+\lambda \mathrm{TV}(I), \quad \lambda>0
$$

where $\operatorname{TV}(I)$ is the discrete TV functional

$$
\operatorname{TV}(I)=\frac{1}{N M} \sum_{i=1}^{N} \sum_{j=1}^{M}\left|\nabla_{i, j} I\right|
$$

and the discrete gradient $\nabla_{i, j} I$ is expressed by means of the forward difference operator as:

$$
\begin{gathered}
\nabla_{i, j} I=\left(\frac{I_{i+1, j}-I_{i, j}}{h_{x}}, \frac{I_{i, j+1}-I_{i, j}}{h_{y}}\right), \quad h_{x}=\frac{1}{N}, \quad h_{y}=\frac{1}{M}, \\
\left|\nabla_{i, j} I\right|=\sqrt{\left(\frac{I_{i+1, j}-I_{i, j}}{h_{x}}\right)^{2}+\left(\frac{I_{i, j+1}-I_{i, j}}{h_{y}}\right)^{2}} .
\end{gathered}
$$

The scalar $\lambda$ is the regularization parameter controlling the tradeoff between the fit to the data and the regularization of the solution. To overcome the difficulties due to the nondifferentiability of the Euclidean norm, TV $(I)$ is usually replaced by the slightly modified functional

$$
\operatorname{TV}(I)=\frac{1}{N M} \sum_{i=1}^{N} \sum_{j=1}^{M}\left|\nabla_{i, j} I\right|_{\beta},
$$

where

$$
\left|\nabla_{i, j} I\right|_{\beta}=\sqrt{\left|\nabla_{i, j} I\right|^{2}+\beta^{2}}
$$

Problem (12) is a strictly convex problem whose well-posedness is proved in Ref. [1]. Its Euler-Lagrange equation, assuming homogeneous Neumann boundary conditions, is

$$
g(I)=\nabla \mathcal{F}(I)=I-I_{\mathrm{d}}(\mathbf{x})+\lambda L_{I, \beta}(I)=0,
$$

where the symmetric positive semidefinite operator $L_{I, \beta}$ is defined as

$$
L_{I, \beta}(R)=\sum_{i=1}^{N} \sum_{j=1}^{M} \nabla_{i, j}^{t}\left(\frac{\nabla_{i, j} R}{\left|\nabla_{i, j} I\right|_{\beta}}\right),
$$

with $\nabla_{i, j}^{t}$ the transpose of $\nabla_{i, j}$. 
The hessian of the objective functional in (12) is given by

$$
H(I)=\mathbf{1}+\lambda L_{I, \beta}+\lambda L_{I, \beta}^{\prime}(I),
$$

where $\mathbf{1}$ is the identity matrix. Further, details on the derivation of the gradient and the hessian of $\mathcal{F}(I)$ can be found in Ref. [13].

To find the solution $I_{\mathrm{TV}}$ of the problem (12) the nonlinear equation (18) is solved by the lagged diffusivity FP algorithm [14,15]. Let $A(I)$ be the positive definite [3,14] operator defined as:

$$
A(I)=\mathbf{1}+\lambda L_{I, \beta} .
$$

The FP method can be described by the iterative procedure:

$$
I^{(k+1)}=I^{(k)}-\left(A\left(I^{(k)}\right)\right)^{-1} g\left(I^{(k)}\right) .
$$

The TV-regularized RIGR algorithm applied to the images of the time sequence is outlined as follows.

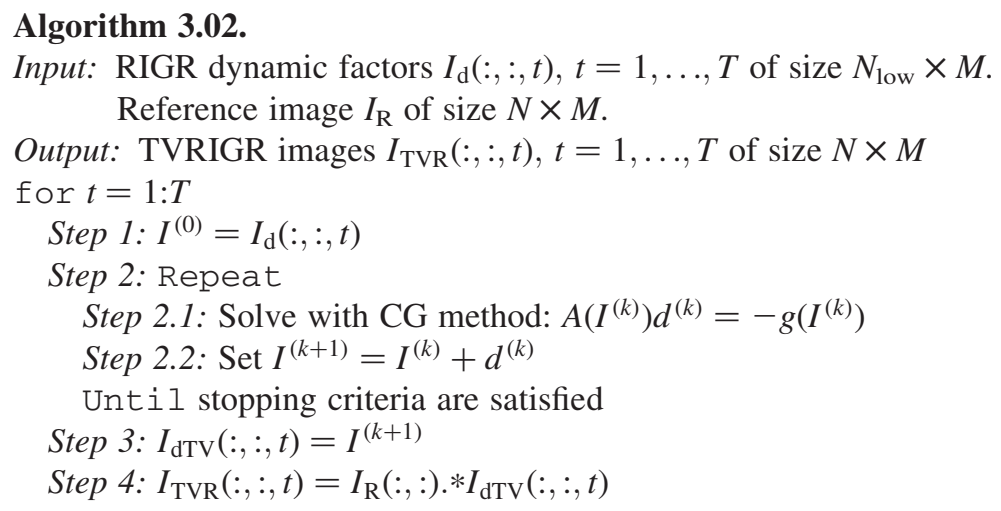

In our implementation, each linear subproblem in step 2.1 is solved by the conjugate gradient (CG) method. These linear systems are highly ill-conditioned because their coefficient matrix $A(I)$ is related to the diffusion coefficient $\left|\nabla_{i, j} I\right|^{-1}$ that can be close to zero, especially when $\beta$ is small, if adjacent pixels have only few differences. For this reason, we use the CG method as a regularization method, by stopping it after few iterations. The iterative procedure in Step 2 is terminated when one of the following stopping criteria is satisfied:

i) $k \geq$ maxit

ii) $\left\|g\left(I^{(k)}\right)\right\|_{2} \leq$ tol $\left\|g\left(I^{(0)}\right)\right\|$

where maxit is a maximum number of iterations and tol is a given tolerance. 


\section{Numerical experiments}

In this section, we report the results obtained with the TV-regularized RIGR method (TVRIGR) applied to simulated and real dynamic MR data. The numerical tests show how the TV regularization acts on dynamic MR images, its effectiveness on noise and artifacts removal. In all the presented test problems, the values of the tolerances of the stopping criteria of algorithm 3.02 are fixed as follows: maximum number of outer FP iterations maxit $=15$; tolerance FP iterations: tol $=0.5$; maximum number of inner CG iteration: 30 (Step 2.1). The quality of the reconstructions is compared by means of the root mean square error (RMSE) defined as:

$$
\mathrm{RMSE}=\sqrt{\frac{1}{M N} \sum_{i=1}^{M} \sum_{j=1}^{N}\left(I_{i j}-I_{i j}^{\text {(exact) })}\right)^{2}}
$$

where the $M \times N$ images $I$ and $I^{\text {(exact) }}$ are the reconstructed and the original images, respectively. Table 1 reports the RMSE parameter obtained by the RIGR and the TVRIGR methods. In all the experiments TVRIGR have always smaller RMSE values. We report the results obtained in the reconstruction of one-dimensional signals (Signal_1 and Signal_2) and in the reconstruction of two images (circle, brain). All the tests require only a few CG and FP iterations. The regularization parameters $(\gamma$ and $\alpha$ ), reported in the figures, are relative to the best reconstructions.

\subsection{Signal_1 test problem}

The first test problem is a 1D simulation consisting of a reference signal $I_{\mathrm{R}}(\mathbf{x})$; (figure 2(a)) and an exact dynamic signal $I^{\text {exact }}(\mathbf{x})$; (figure 2(b)), both of $N=256$ samples. The noisy under-sampled dynamic data are obtained by adding Gaussian white noise to the exact dynamic signal and considering only a subset $\tilde{D}(\mathbf{k})$ with the $N_{\text {low }}=64$ symmetric lowest frequencies. The amount of noise measured in this test problem is signal to noise ratio $(\mathrm{SNR}=78 \mathrm{~dB})$. The dynamic signals, reconstructed with the RIGR and the TVRIGR methods, are depicted in figure 2(c) and (d), respectively. As we expected the TVRIGR greatly reduces the oscillations in the reconstructed signal.

\subsection{Signal_2 test problem}

This test problem consists of a reference signal $I_{\mathrm{R}}(\mathbf{x})$; (figure 3(a)) and an exact dynamic signal $I^{\text {exact }}(\mathbf{x})$; (figure $3(\mathrm{~b})$ ), both of $N=256$ samples. The under-sampled dynamic data are

Table 1. Error parameters for test problems with simulated MR data.

\begin{tabular}{llc}
\hline Test problem & Method & RMSE \\
\hline Signal_1 & RIGR & $1.171224 \times 10^{-2}$ \\
& TVRIGR & $1.134237 \times 10^{-2}$ \\
Signal_2 & RIGR & $2.639253 \times 10^{-2}$ \\
Circle & TVRIGR & $2.456414 \times 10^{-2}$ \\
& RIGR & $1.880189 \times 10^{-2}$ \\
& TVRIGR & $1.807084 \times 10^{-2}$ \\
\hline
\end{tabular}


(a)

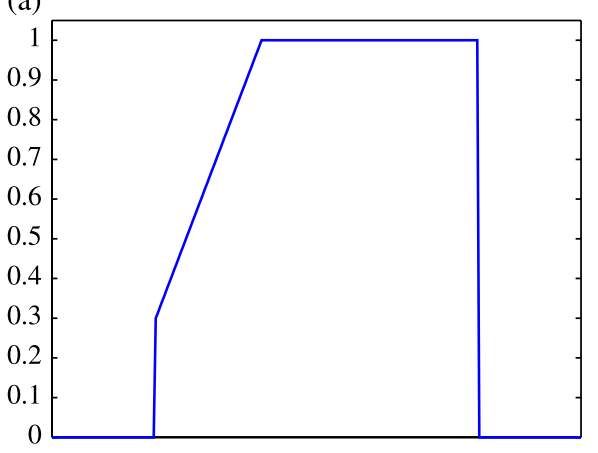

(c)

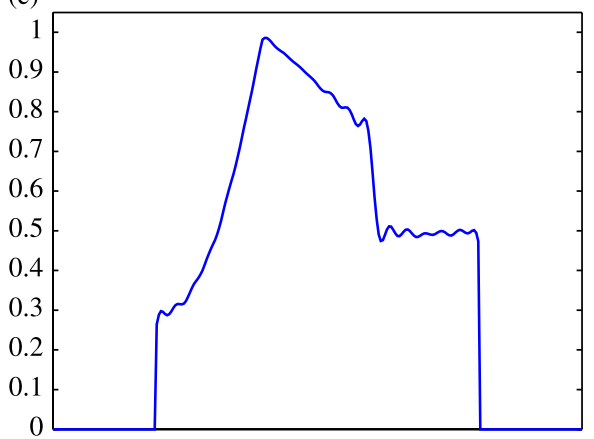

(b)
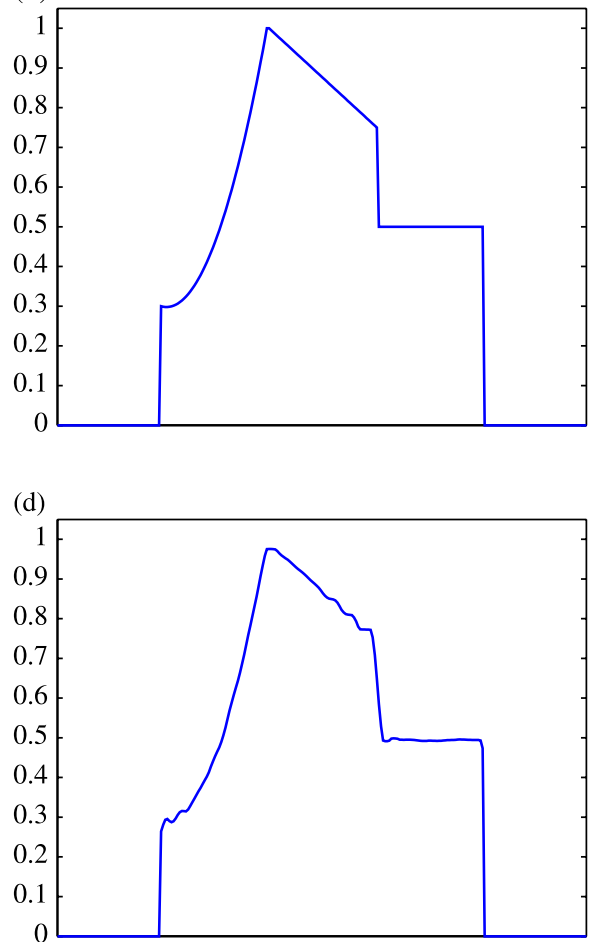

Figure 2. Test problem: signal_1; (a) reference signal; (b) dynamic signal; (c) RIGR $\left(\gamma=10^{-2}\right.$ ); (d) TVRIGR $\left(\alpha=10^{-4}\right)$.

obtained by considering only a subset $\tilde{D}(\mathbf{k})$ with the $N_{\text {low }}=64$ symmetric lowest frequencies of the spectrum of $I^{\text {exact }}(\mathbf{x})$.

The dynamic signals reconstructed with the RIGR and the TVRIGR methods are depicted in figure 3(c) and (d), respectively. The TV reconstruction is optimal for this test problem, and the values of RMSE reported in table 1 confirm the analysis. For this test problem $\gamma=5 \times 10^{-5}$.

\subsection{Circle test problem}

This test problem is the so-called circle test problem and it is used in the literature to represent the action of a contrast agent in the examined organ through the variations of gray levels in the dynamic image. Two images of size $256 \times 256$ represent the reference image $I_{\mathrm{R}}(\mathbf{x}, \mathbf{y})$ and the exact dynamic image $I^{\text {exact }}(\mathbf{x}, \mathbf{y})$, respectively (figure 4). After Fourier transforming $I^{\text {exact }}(\mathbf{x}, \mathbf{y})$, a reduced scan spin-echo acquisition is simulated by considering the $64 \times 256\left(N_{\text {low }}=64\right)$ dynamic lowest frequencies. White noise with $\mathrm{SNR} \simeq 61 \mathrm{~dB}$ has been added to the $N_{\text {low }} \times 256$ available dynamic frequencies. Figure 5(a) and (b) show the reconstructions obtained with the RIGR and the TVTIGR methods, respectively. The Gibbs artifacts in the RIGR reconstruction are clearly shown in figure 6(a) where the error image $I^{\text {exact }}(\mathbf{x}, \mathbf{y})-I^{\text {RIGR }}(\mathbf{x}, \mathbf{y})$ is represented. We can observe from figure 6(b) that represents the error image $I^{\text {exact }}(\mathbf{x}, \mathbf{y})-I^{\text {TVRIGR }}(\mathbf{x}, \mathbf{y})$ that the artifacts are considerably reduced. The RMSE measures (table 1) confirm these considerations. 
(a)

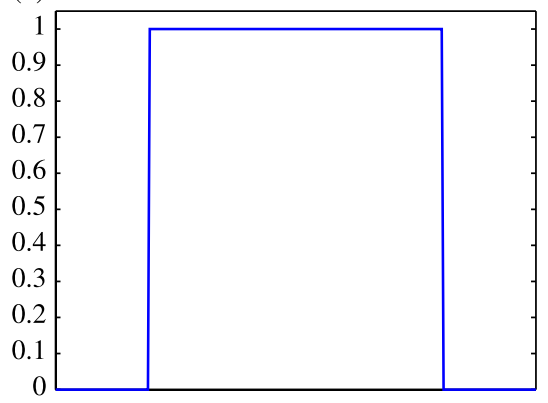

(c)

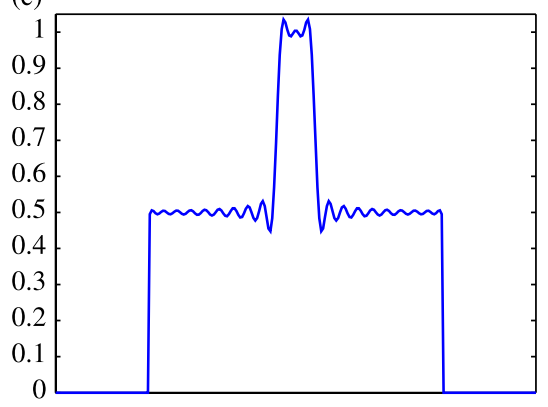

(b)

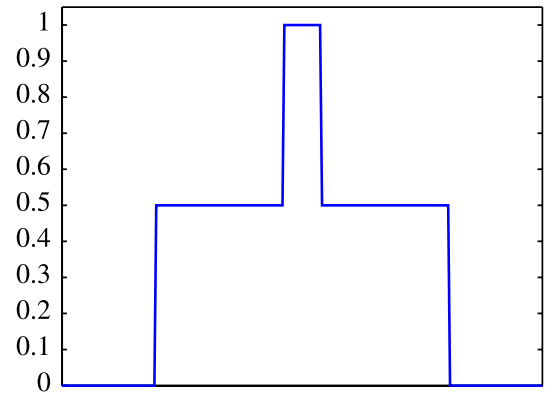

(d)

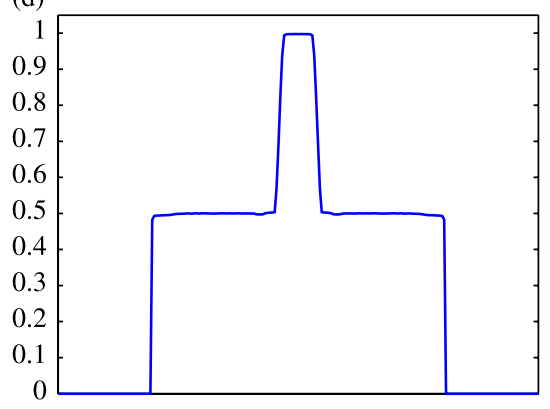

Figure 3. Test problem: signal_2; (a) reference signal; (b) dynamic signal; (c) RIGR $\left(\gamma=10^{-2}\right.$ ); (d) TVRIGR $\left(\alpha=5 \times 10^{-4}\right)$.

(a)

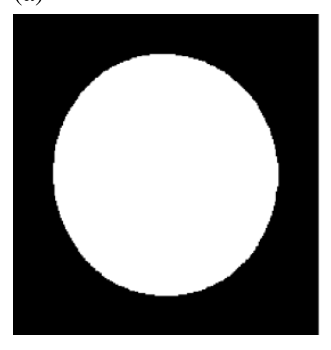

(b)

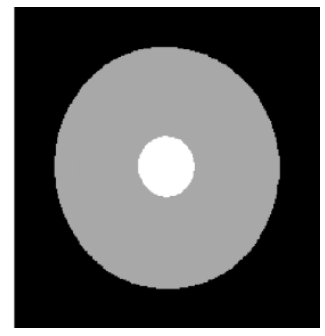

Figure 4. Test problem: circle; (a) reference image; (b) dynamic image.

(a)

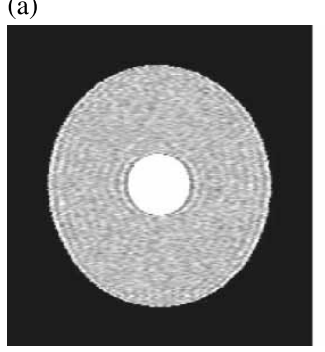

(b)

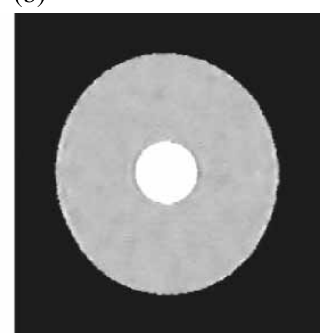

Figure 5. Test problem circle with noise; RIGR $\left(\gamma=5 \times 10^{-2}\right)$; (b) TVRIGR $(\alpha=5)$. 
(a)

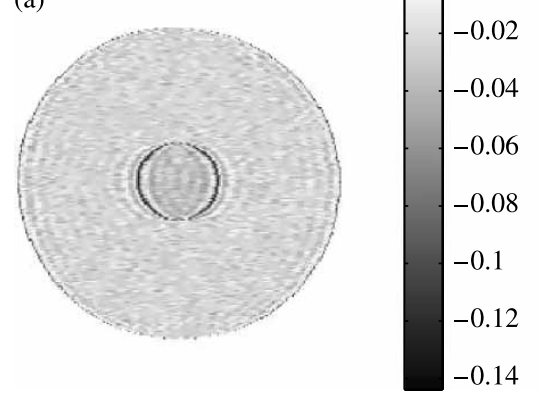

(b)

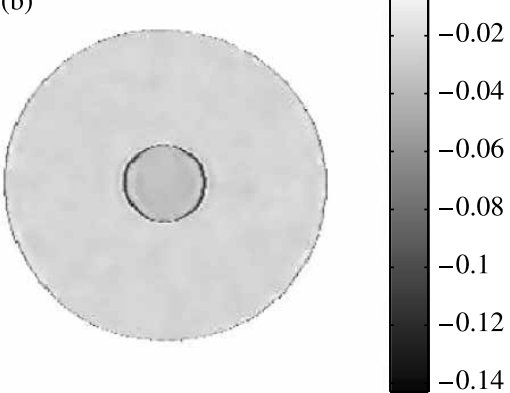

Figure 6. Test problem circle with noise, error images; (a) RIGR; (b) TVRIGR.

\subsection{Brain test problem: real MR data}

The real MR data of the brain test problem are constituted of 58 data sets from a human brain: a baseline reference data set $D_{\mathrm{R}}\left(\mathbf{k}_{x}, \mathbf{k}_{y}\right)$ and 57 low-sampled dynamic data sets of $19 \times 128$ samples, acquired by a MR spin-echo technique after injecting a contrast agent. The reference image $I_{R}(\mathbf{x}, \mathbf{y})$ is shown in figure $7(a)$. Figure 7(b) and (c) show the reconstructions of slice 26 obtained with the RIGR and the TVTIGR methods, respectively. The differences between the RIGR and TVRIGR reconstructions become more evident in the representation of the dynamic multiplicative factors (figure 8). Comparing the images reported in figure 8(a) and (b) it is evident in the attenuation of the Gibbs artifacts in the TVRIGR reconstruction.

(a)

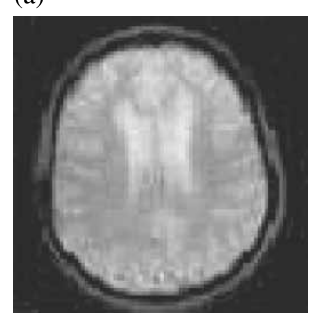

(b)

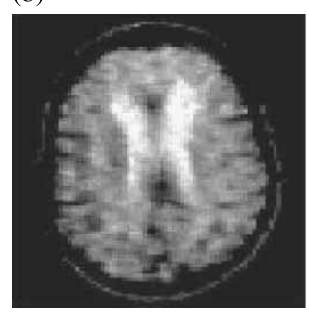

(c)

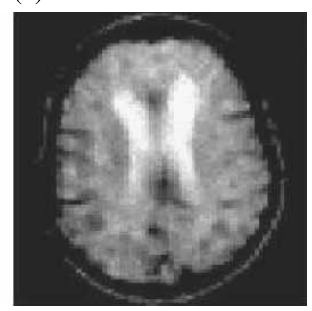

Figure 7. Test problem: brain; (a) reference image; (b) RIGR $(\gamma=0)$; (c) TVRIGR $(\alpha=5)$.

(a)

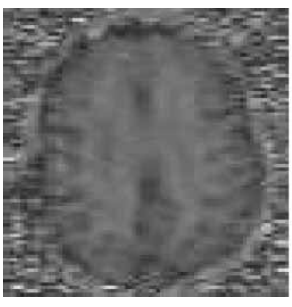

(b)

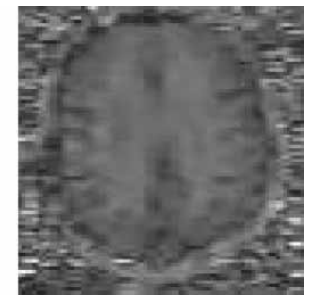

Figure 8. Test problem brain, reconstructions of the dynamic factor with the RIGR and TVRIGR methods; (a) RIGR dynamic; (b) TVRIGR dynamic factor. 


\section{Conclusions}

In this paper, we have applied the TV method for regularizing the reconstruction of limited resolution data in the Fourier domain. The ringing artifacts that affect the image reconstructed with the generalized series approach (RIGR method) are considerably reduced by the TV regularization.

\section{Acknowledgements}

This work was supported by the Italian Ministero dell' Università e della Ricerca (MIUR) project Inverse Problems in Medical Imaging 2004-2006 (Grant No. 2004015818).

\section{References}

[1] Acar, R. and Vogel, R.C., 1994, Analysis of total variation penalty methods for ill-posed problems, Inverse Problems, 10, 1217-1229.

[2] Bronstein, M., Bronstein, A. and Zibulevsky, M., 2002, Reconstruction in diffraction ultrasound tomography using non-uniform FFT, IEEE Transactions on Medical Imaging, 21(11), 1395-1401.

[3] Chan, T.F. and Mulet, P., 1999, On the convergence of the lagged diffusivity fixed point method in total variation image restoration, SIAM Journal on Numerical Analysis, 36(2), 354-367.

[4] Jonsson, E., Huang, S.C. and Chan, T., 1998, Total variation regularization in positron emission tomography. Technical Report CAM 98-48, UCLA.

[5] Keeling, S.L., 2003, Total variation based convex filters for medical imaging, Applied Mathematics and Computation, 139, 101-119.

[6] Landi, G. and Loli Piccolomini, E., Numerical methods for the reconstruction of dynamic magnetic resonance images. To apper on Inverse Problems in Science and Engineering.

[7] Landi, G. and Loli Piccolomini, E., 2005, A total variation regularization strategy in dynamic MRI, Optimization Methods and Software, 20(4-5), 545-558.

[8] Landi, G. and Loli Piccolomini, E., 2005, Numerical methods for dynamic magnetic resonance imaging. Technical report, Almae Matris Studiorum Acta, August.

[9] Landi, G., Piccolomini, E.L. and Zama, F., 2004, A total variation-based regularization strategy in magnetic resonance imaging. In: R.P. Millane, P.J. Bones and M.A. Fiddy (Eds) Image Reconstruction from Incomplete Data III, Proceedings of SPIE, 5562, pp. 141-151.

[10] Liang, Z-P. and Lauterbur, P.C., 1994, An efficient method for dynamic magnetic resonance imaging, IEEE Transactions on Medical Imaging, 13(4), 677-686.

[11] Rudin, L.I., Osher, S. and Fatemi, E., 1992, Nonlinear total variation based noise removal algorithms, Physica $D, \mathbf{6 0}, 259-268$.

[12] Teboul, S., Blanc-Féraud, L., Aubert, G. and Barlaud, M., 1998, Variational approach for edge-preserving regularization using coupled PDE's, IEEE Transactions on Image Processing, 7, 387-397.

[13] Vogel, C.R., 2002, Computational Methods for Inverse Problems (Philadelphia, PA: SIAM).

[14] Vogel, C.R. and Oman, M.E., 1996, Iterative methods for total variation denoising, SIAM Journal on Scientific Computing, 17, 227-238.

[15] Vogel, C.R. and Oman, M.E., 1998, Fast, robust total variation-based reconstruction of noisy, blurred images, IEEE Transactions on Image Processing, 7, 813-824. 


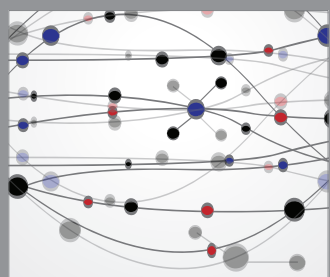

The Scientific World Journal
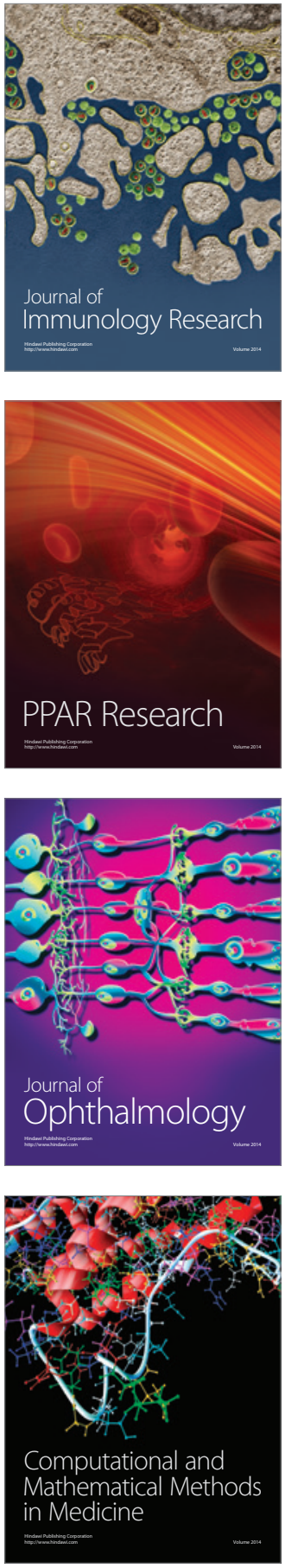

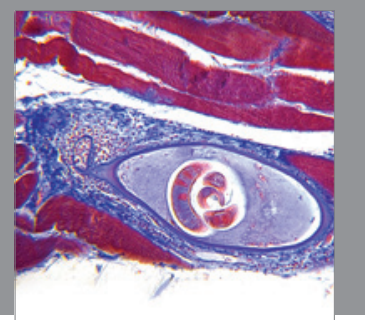

Gastroenterology

Research and Practice
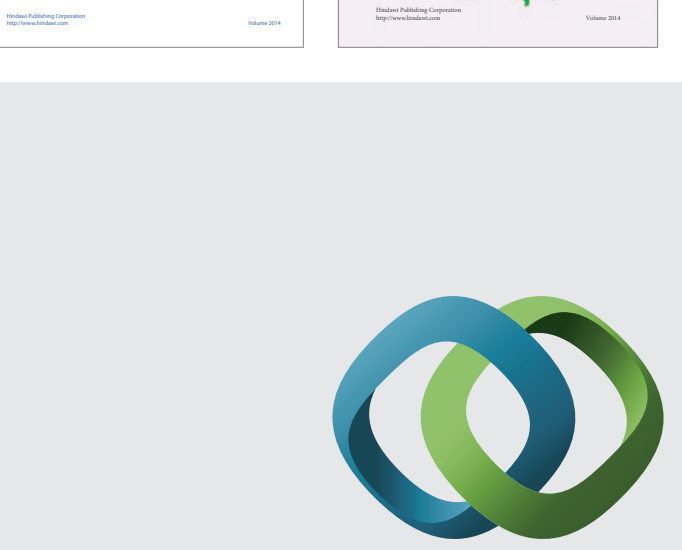

\section{Hindawi}

Submit your manuscripts at

http://www.hindawi.com
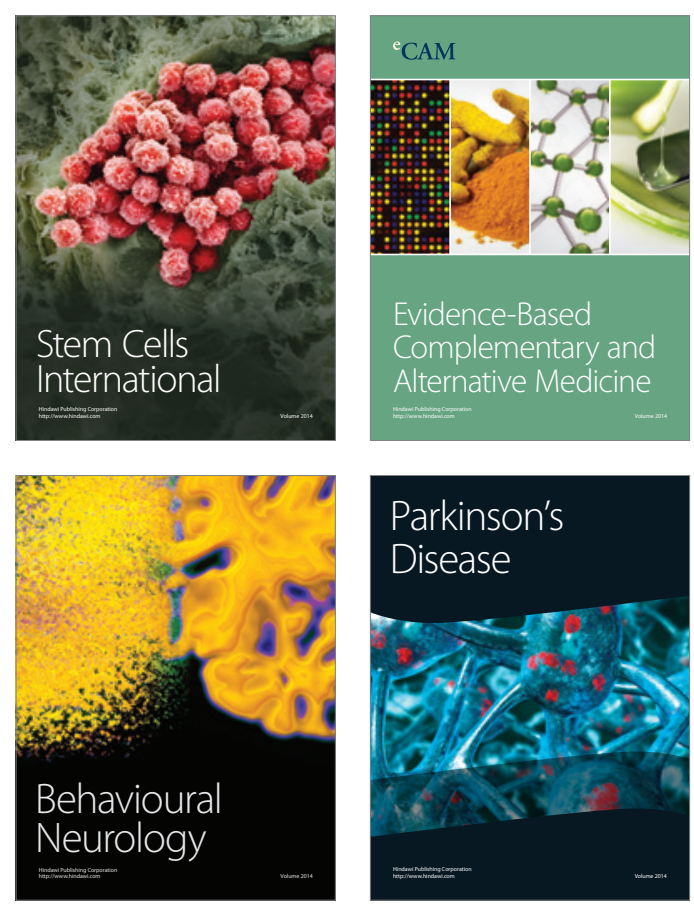

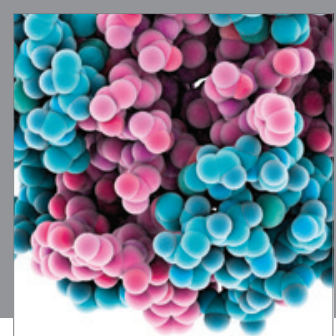

Journal of
Diabetes Research

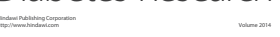

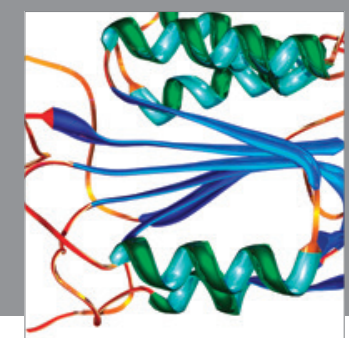

Disease Markers
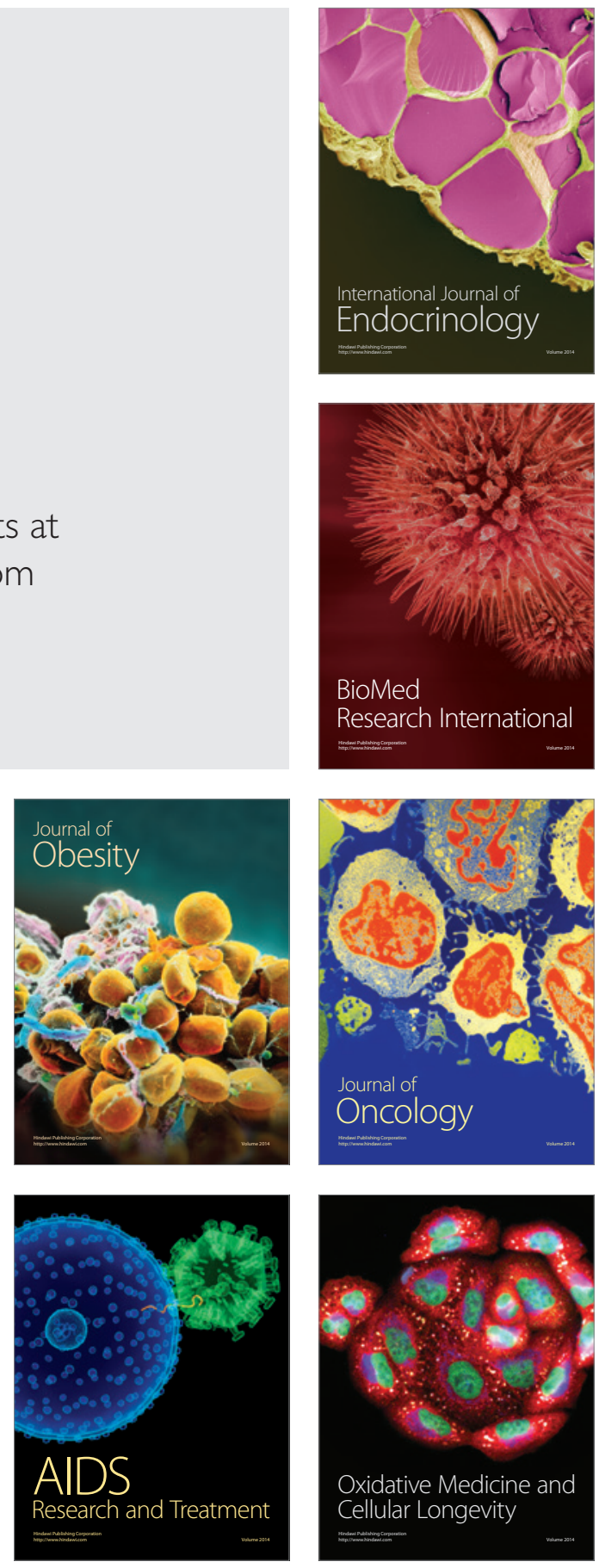